\title{
Estudio comparativo de dismenorrea en dos colegios de Bogotá de clase social diferente
}

\author{
William Onatra H.*; Pedro Martínez**; Germán Franco***; Gonzalo Vargas P.****
}

RESUMEN OBJETIVO: Conocer si hay variaciones epidemiológicas de la dismenorrea en dos clases sociales en la ciudad de Santafé de Bogotá. Colombia.

MATERIAL Y METODOS: Se realiza una encuesta en dos colegios de Bogotá, colegio A No. 496 y colegio B No. 307 sobre dolor de acuerdo con la clasificación internacional y se realizan las pruebas de significancia CHI 2 y la diferencia de promedios.

RESULTADOS: El promedio de edad de la menarquia del colegio A fue 12.1 años y del $B 12.8$ años con una $P<0.01$ para $A / B$. La frecuencia y duración de la menstruación fue de 28.6x5 días para el colegio A y de 28.8x4.5 para el colegio B sin significancia estadística para la frecuencia y una $\mathbf{P}<0.01$ en la duración mayor en A que B. La frecuencia del dolor en general fue de $75.9 \%$ (A)/s75.0\% (B) sin significancia estadística.

El grado de dolor según la clasificación demostró:

\begin{tabular}{|c|c|c|c|c|c|c|c|}
\hline \multirow[b]{2}{*}{ Grados } & \multicolumn{2}{|c|}{ Colegio A } & \multicolumn{2}{|c|}{ Colegio B } & \multicolumn{2}{|c|}{ Total } & \multirow[t]{2}{*}{ Sig/Estadis. } \\
\hline & $\mathbf{n}$ & $\%$ & $\mathrm{n}$ & $\%$ & $\mathbf{n}$ & $\%$ & \\
\hline 0 & 172 & 34.7 & 27 & 41.1 & 299 & 37.2 & $\mathrm{P}<0.1 \mathrm{~B} / \mathrm{A}$ \\
\hline I & 214 & 43.1 & 113 & 36.8 & 327 & 40.7 & $\mathrm{P}<0.1 \mathrm{~A} / \mathrm{B}$ \\
\hline II & 68 & 13.7 & 36 & 11.7 & 104 & 12.9 & NS \\
\hline III & 14 & 2.8 & 19 & 6.1 & 33 & 4.1 & $\mathrm{P}<0.5 \mathrm{~B} / \mathrm{A}$ \\
\hline NR & 28 & 5.6 & 12 & 3.9 & 40 & 5.0 & \\
\hline Total & 496 & & 307 & & 803 & & \\
\hline
\end{tabular}

CONCLUSION: Esta encuesta en adolescentes demuestra una clara diferencia de los grados de dolor mayor en el colegio A que en el B, planteando nuevos interrogantes sobre la dismenorrea.

PALABRAS CLAVES: Dismenorrea, adolescencia.

SUMMARY OBJECTIVE: To know if there are epidemiological differences in two schools at Bogota from different social class in relation with dismenorrea.

MATERIAL AND METHODS: Asurvey was performed in two schools of Bogota. In the school A. 496 patients and in B 307 patients The survey is about pain in accondance with the international classification and it was made the CHI 2 and the difference in arrage.

RESULTS: The arrage age fon the menarche in group A. was 12.1 years old and 12.3 in $\mathrm{B}$. With an $\mathrm{p}<0.01$ fon $\mathrm{A} / \mathrm{B}$.

The frecuency and duration of the period was $28 \times 5$ days for group. A and $28.8 \times 4.5$ for group B without statistical difference: for the frecuency and $A P<0.01$ in the duration, higer in $A$ thaw in $B$.

The frecuency for pain in general was $75.9 \% \mathrm{~A}$ and $75 \% \mathrm{~B}$ with out statistical significance.

The grade of pain in relation with the international classification shows.

CONCLUSION: This survey shows that there is a clear differences between the grade of pain from college $A$ and $B$, showing collegue $A$ with a higher grade of pain from B; creating a new question about dismenorrea.

KEY WORDS: Dysmenorrhea, adolescents.

* Profesor Asistente Departamento de Obstetricia y Ginecología. Universidad Nacional de Colombia. Unidad de Endocrinología Ginecología. Instituto Materno Infantil. Bogotá.

** Jefe Departamento Clínico Cafam. Unidad de Adolescencia.

*** Jefe de Investigaciones y Medicina Preventiva Cafam.

**** Profesor Asistente. Departamento de Medicina Preventiva. Universidad Nacional de Colombia.

\section{Introducción}

La dismenorrea es una de las entidades que prevalece en un $50 \%$ durante la adolescencia. En la primera encuesta publicada en nuestro medio mostró que su frecuencia era del $75.3 \%$ (1). La dismenorrea severa (Grado III) se describe entre un 10-15\% para países nórdicos (2-3) contrastando con datos latinoamericanos de un 3-5\% $(1,4)$. Según cifras norteamericanas por ausentismo laboral y dismenorrea se 
pierden unos 600 millones de horas de trabajo anual (5). Adicionalmente se describen las alteraciones personales y familiares de esta entidad. La dismenorrea se presenta con mayor frecuencia entre los 15 y 24 años disminuyendo al aumentar la edad (6) sinembargo los autores demostraron que el dolor es mayor al iniciar la menarquia para descender progresivamente con la edad (1).

Diferentes estudios demuestran que desde el punto de vista epidemiológico fuera de disminuir con la edad su incidencia es mayor en solteras que casadas y mayor en las mujeres que trabajan fuera del hogar frente a las que permanecen en él (7). Estos puntos de vista sugieren que el «stress» puede contribuir en la percepción de los niveles de dolor pero la incapacidad que genera la dismenorrea no depende del tipo de oficio o trabajo porque se ha demostrado la disminución en la eficiencia laboral o de estudio durante los períodos de dolor independiente del tipo de ocupación (8).

Con el progreso en las determinaciones hormonales y el descubrimiento de las prostaglandinas (9), la utilidad de los antiinflamatorios no esteroides (AINES) (10) y anticonceptivos (11) en el manejo de la dismenorrea plantearon un nuevo enfoque diagnóstico y terapéutico. Estos planteamientos determinaron que los aspectos psicoemocionales tan en boga antes de los años 60 y pasarán a un segundo plano. Un punto importante ha sido la parte cultural y su comportamiento en el área rural y urbana, en este último no se ha realizado estudio en nuestro medio. A nivel de clase socioeconómica los estudios sobre el tema son contradictorios: (13-14) se postula que su incidencia podría ser mayor en la clase alta en parte por actitudes aprendidas de la madre, acceso a mayor información, mejor alimentación, mayor cantidad de sustrato de colesterol, aumento de prostaglandinas, menor cantidad de betaendorfinas. Si el dolor se presenta ¿Cuáles serían las medidas terapéuticas utilizadas? Estos interrogantes llevaron a los autores a realizar esta encuesta comparativa.

\section{Material y métodos}

Se realiza una encuesta comparativa en dos colegios de Santafé de Bogotá de diferente clase social entre adolescentes de 10 a 18 años. La encuesta incluía edad actual, edad de la menarquia, frecuencia y duración de los ciclos, frecuencia del dolor, dolor pre, durante o postmenstrual, clasificación del dolor según los grados: grado $\mathrm{O}=$ no dolor, grado $\mathrm{I}=$ leve, Grado $\mathrm{II}=$ moderado, Grado $\mathrm{III}=$ severo. Para esta clasificación se tuvieron los siguientes parámetros: A) si el dolor se acompañaba de cefalea, náusea, vómito, calambres. B) si era incapacitante o no. C) si era necesario tomar alguna medicación. Por último se preguntaba qué medidas terapéuticas se utilizaban para mejoría del dolor.

Para comparar estas dos encuestas se utilizó la prueba de Chi 2, la diferencia de proporciones y de promedios. El análisis de varianza hace comparable estos dos grupos poblacionales.

\section{Resultados}

Como lo muestra la tabla 1 el total de las adolescentes encuestadas es de 766 , colegio A clase socioeconómica alta: 481 y colegio B clase socioeconómica media-baja: 285.
Tabla 1

COLEGIOSPARTICIPANTES

\begin{tabular}{|cll|}
\hline Colegio & $\mathbf{n}$ & \% \\
\hline A & 481 & 61.7 \\
B & 285 & 38.3 \\
\hline Total & $\mathbf{7 6 6}$ & $\mathbf{1 0 0 . 0}$ \\
\hline
\end{tabular}

El promedio de la menarquia según la edad para $\mathrm{A}$ fue de 12.1 y para $B$ de 12.8 con clara diferencia significativa $(\mathrm{P}<0.01)$ para A/B. Tabla 2.

Tabla 2

EDAD PROMEDIO DE LA MENARQUIA

\begin{tabular}{|c|c|c|c|c|}
\hline \multirow[b]{2}{*}{ Años } & \multicolumn{3}{|c|}{ COLEGIOS } & \\
\hline & $\mathbf{n}$ & $\%$ & $\mathbf{n}$ & $\%$ \\
\hline 9 & 10.2 & & 1 & 0.4 \\
\hline 10 & 29 & 5.0 & - & - \\
\hline 11 & 104 & 21.0 & 36 & 12.6 \\
\hline 12 & 166 & 33.5 & 88 & 30.9 \\
\hline 13 & 148 & 29.8 & 95 & 33.3 \\
\hline 14 & 26 & 5.2 & 47 & 16.5 \\
\hline 15 & 7 & 1.4 & 12 & 4.2 \\
\hline 16 & - & - & 3 & 1.1 \\
\hline 17 & - & - & 2 & 0.7 \\
\hline 19 & - & - & 1 & 0.4 \\
\hline NR & 15 & 3.0 & 22 & 7.1 \\
\hline
\end{tabular}

\begin{tabular}{|lll|}
\hline \multicolumn{3}{|c|}{ PRUEBA ESTADISTICA } \\
Colegio & \multicolumn{1}{c|}{ A } & B \\
\hline $\mathrm{n}$ & 481 & 481 \\
Edad promedio & 12.1 & 12.8 \\
D.S. & 1.06 & 1.22 \\
S2 & 1.12 & 1.48 \\
COEF.VIZA & $8.8 \%$ & $9.5 \%$ \\
Zc & \multicolumn{2}{|c|}{8.05} \\
P & \multicolumn{2}{|c|}{$\mathrm{P}<0.01$} \\
\hline
\end{tabular}

La edad de la menarquia es menor en A que en B.

Al comparar la frecuencia y duración de la menstruación por tablas 3 y 4 , no hubo diferencia en cuanto a la frecuencia pero si la duración es significativamente mayor en A que en $\mathrm{B}(\mathrm{P}<0.01)$.

Tabla 3

COMPARATIVO DE LA FRECUENCIA DE LA MENSTRUACION

\begin{tabular}{|lrrr|}
\hline Colegio & \multicolumn{1}{c|}{ A } & \multicolumn{1}{c|}{ B } \\
\hline n & 481 & & 281 \\
Promedio & 28.5 & & 28.7 \\
& & & \\
S2 & 9.6 & & 7.9 \\
COEF.V/ZA & $10.9 \%$ & & $9.8 \%$ \\
Zc & & 0.91 & \\
P.SIG. & & NS. & \\
\hline
\end{tabular}

No hay significancia en cuanto a la frecuencia menstrual. 
Tabla 4

DURACION DE LA MENSTRUACION

\begin{tabular}{|c|c|c|}
\hline Colegio & A & B \\
\hline $\mathrm{n}$ & 476 & 233 \\
\hline $\mathrm{X}$ Días & 5 & 4 \\
\hline DS & 1.47 & 1.53 \\
\hline S2 & 2.15 & 2.33 \\
\hline COEF.V/ZA & $29 \%$ & $34 \%$ \\
\hline $\mathrm{Zc}$ & & \\
\hline PSIG. & & \\
\hline
\end{tabular}

La duración es mayor en A que en B.

A la pregunta general si había o no dolor durante la menstruación, no hubo diferencia significativa en los dos grupos con un porcentaje igual de dolor en $75 \%$. Tabla 5.

Tabla 5

DOLOR EN GENERAL DURANTE LA MENSTRUACION

\begin{tabular}{|cccc|}
\hline Colegio & A & B \\
\hline $\mathrm{n}$ & 481 & & 285 \\
$\mathrm{X}$ & 75.9 & & 75.0 \\
$\mathrm{Zc}$ & & 0.28 & \\
$\mathrm{PS}$. & & NS & \\
\hline
\end{tabular}

No hay diferencia significativa.

Según los grados de dolor y al hacer las pruebas de significancia el grado 0 o no dolor es mayor en colegio A que el B. $(\mathrm{P}<0.01)$. El grado leve es mayor en A que en B $(\mathrm{P}<0.01)$. El grado II (moderado) no hubo diferencia y en el grado III (severo) es mayor en el colegio B/A. $(\mathrm{P}<0.05)$. Tabla 6.

Tabla 6

DOLOR SEGUN LOS GRADOS

\begin{tabular}{|lrrrrrr|}
\hline \multicolumn{2}{|l}{ Colegio } & A & \multicolumn{2}{c}{ B } & \multicolumn{2}{c|}{ Total } \\
\hline \multicolumn{2}{|l}{ Grados } & \multicolumn{1}{c}{ \% } & \multicolumn{1}{c|}{ n } & \multicolumn{1}{c|}{$\%$} & \multicolumn{1}{c|}{ n } & \% \\
\hline 0 & 172 & 34.7 & 127 & 41.4 & 299 & 37.2 \\
I & 214 & 43.1 & 113 & 36.8 & 327 & 40.7 \\
II & 68 & 13.7 & 36 & 11.7 & 104 & 12.9 \\
III & 14 & 2.8 & 19 & 6.2 & 33 & 4.1 \\
NR & 28 & 5.6 & 12 & 3.9 & 40 & 5.0 \\
\hline
\end{tabular}

PRUEBAS DE SIGNIFICANCIA ESTADISTICA

$\begin{array}{lrrr}\text { Grado 0 } & \text { SIG } & & \\ \text { Grado I } & 10 \% & 1.89 & \mathrm{~B}>\mathrm{A} \\ \text { Grado II } & 10 \% & 1.78 & \mathrm{~A}>\mathrm{B} \\ \text { Grado III } & \text { NS } & 0.83 & = \\ & 5 \% & 2.17 & \mathrm{~A}>\mathrm{B}\end{array}$

En relación con la terapéutica la tabla 7 no muestra predilección por ningún medicamento exceptuando la hioscimina llamando la atención que aquellas adolescentes del grupo 0 o de no dolor toman alguna medicación durante la menstruación.

Tabla 7

MEDICAMENTOS UTILIZADOS SEGUN EL GRADO DE DOLOR

\begin{tabular}{|c|c|c|c|c|c|c|c|c|c|c|c|c|c|c|c|c|c|c|c|c|}
\hline Grupos & 0 & & & & I & & & & II & & & & III & & & & Tot & & & \\
\hline Colegio & A & & B & & $\mathrm{A}$ & & B & & A & & B & & A & & B & & A & & B & \\
\hline Fármaco & $\mathrm{n}$ & $\%$ & $\mathrm{n}$ & $\%$ & $\mathrm{n}$ & $\%$ & $\mathrm{n}$ & $\%$ & $\mathrm{n}$ & $\%$ & $\mathrm{n}$ & $\%$ & $\mathrm{n}$ & $\%$ & $\mathrm{n}$ & $\%$ & $\mathrm{n}$ & $\%$ & $\mathrm{n}$ & $\%$ \\
\hline ASA & 2 & 0.4 & & & 10 & 2.0 & & & 5 & 1.0 & 1 & 0.3 & & & & & 17 & 3.4 & 1 & 0.3 \\
\hline A/Nofen & 1 & 0.2 & & & 6 & 1.2 & & & 6 & 1.2 & 1 & 0.3 & 3 & 0.6 & 1 & 0.3 & 16 & 3.2 & 2 & 0.6 \\
\hline A.Mefen & & & 2 & 0.6 & 2 & 0.4 & 6 & 1.9 & & & 3 & 0.9 & 3 & 0.6 & 5 & 1.6 & 5 & 1.0 & 1 & 65.2 \\
\hline B.Hiosc & 9 & 1.8 & 4 & 1.3 & 27 & 5.4 & 22 & 7.1 & 20 & 4.0 & 15 & 4.8 & 4 & 0.8 & 11 & 3.5 & 60 & 12 & 52 & 16.9 \\
\hline D/Rona & 1 & 0.2 & & & & & 3. & 09 & & & 1 & 0.3 & & & 1 & 0.3 & 1 & 0.2 & 5 & 1.6 \\
\hline P/Can & 1 & 0.2 & 1 & 0.3 & 6 & 1.2 & 1 & 0.3 & 4 & 0.8 & 2 & 0.6 & 2 & 0.4 & & & 13 & 2.6 & 4 & 1.3 \\
\hline I/Profen & & & & & 3 & 0.6 & & & 1 & 0.2 & & & & & & & 4 & 0.8 & & \\
\hline N.R & & & 2 & 0.6 & & & 1 & 0.3 & & & 3. & 09 & & & 1 & 0.3 & 2 & 0.4 & 7 & 2.2 \\
\hline Subtotal & 14 & 2.8 & 9 & 2.9 & 54 & 10.8 & 33 & 10.7 & 36 & 7.2 & 26 & 8.4 & 12 & 2.4 & 19 & 6.1 & & & & \\
\hline Sin Tto. & 156 & 31.4 & 160 & 32.2 & 32 & 6.4 & 10 & 3.2 & 2 & 0.4 & 378 & 76.2 & 220 & 71.6 & 118 & 38.4 & 80 & 26.0 & & \\
\hline NR & & & & & & & & & & & & & & & & & 28 & 5.6 & 12 & 3.9 \\
\hline TOTAL & & & & & & & & & & & & & & & & & 496 & & 307 & \\
\hline
\end{tabular}




\section{Discusión}

Este estudio pretendía confirmar si los datos de la primera encuesta estaban de acuerdo con los parámetros de menarquia, ciclos, duración, prevalencia del dolor, los grados de dolor y la terapéutica utilizada.

Los datos obtenidos en este estudio comparativo muestran que la menarquia en estas dos poblaciones de diferente clase social permanece dentro de los promedios encontrados en la encuesta nacional para Colombia (13.6 años) (15) y de 12.5 años en una escuela americana (16). Al realizar las pruebas de significancia estadística sobre la edad de la menarquia esta es más temprana en el colegio A que en el B y donde podríamos plantear la hipótesis de un mayor aporte de nutrientes y por lo tanto mayor peso, mayor contenido de grasa explicaría esta diferencia pero no se hizo la encuesta nutricional para confirmar este hecho. Si bien la frecuencia y duración de la menstruación permanece dentro de los límites internacionales ( 28 × 3-4 días) contradice los hallazgos de una encuesta de la OMS en pacientes adolescentes donde la duración post-menarquia fue de 34.1 en el primer año y 31.7 en el segundo año (17). La duración de la menstruación presenta una diferencia significativa y plantea de nuevo un interrogante para el cual no tenemos una explicación satisfactoria.

Al examinar la prevalencia del dolor $75.9 / 75.0$ que confirma los hallazgos iniciales que la dismenorrea en nuestro medio es de este valor. Al comparar de nuevo otros datos nos encontramos que los trabajos de Sudell informa $67 \%$ (17), Lee y col. 60\% (19) Barros y col. 76.3\% (24) y Bjorn $72.4 \%$ (13).

Los datos sobre los grados de dolor demuestran significativamente que el dolor es mayor en clase social alta $65.3 \%$ (colegio A) que en colegio B (58.9\%). Una explicación a este fenómeno tendría una hipótesis alimentaria donde la mayor ingesta de proteínas y grasas nos pondrían una mayor disponibilidad de ácidos grasos (fosfolípidos) sustrato importante para producir por vía de la lipooxigenasa los leucotrienos y por vía ciclopooxigenasa prostaciclina, prostaglandinas y tromboxano A. $(14,26,28)$. Por otra parte se sabe que el «stress» juega un papel importante en la dismenorrea (18), donde el ejercicio la mejora (20) quedando el interrogante el papel de estos dos eventos en la población estudiada. El dolor leve (Grado I) y severo (Grado III) muestran diferencias significativas llamando la atención que el dolor severo e incapacitante en nuestro medio contradice los hallazgos de otros investigadores donde éste tiene cifras del 45.6\% (22), 23\% (23), 44\% (27), $43 \%$ (30) y está de acuerdo con los datos del Brasil que son de $8 \%$ (24), $5.7 \%$ (17). Tabla 8.

Tabla 8

ESTUDIOS COMPARATIVOS SEGUN EL GRADO DE DOLOR

\begin{tabular}{|c|c|c|c|}
\hline Grados & $\begin{array}{l}\text { Wilson (17) } \\
\%\end{array}$ & $\begin{array}{l}\text { Barros (24) } \\
\%\end{array}$ & $\begin{array}{l}\text { Onatra } \\
\%\end{array}$ \\
\hline 0 & 47.5 & 23.6 & 37.2 \\
\hline I & 31.8 & 40.4 & 40.7 \\
\hline II & 15.0 & 27.8 & 12.9 \\
\hline III & 5.7 & 8.2 & 4.1 \\
\hline
\end{tabular}

En revisión de la literatura nos muestran cifras escandalosas sobre las horas de trabajo por incapacidad de la dismenorrea sin embargo a nivel tropical parece que esta cifra no es de esa magnitud y valdría la pena investigar en nuestro medio este tipo de información en la mujer trabajadora. Sobre este tema se plantean otras hipótesis como la refractariedad del dolor de nuestra mezcla racial (25) o el medio ambiente tropical por temperatura, luz, menor stress $(18,20)$.

Terapéuticamente es claro que en este medio escolar los remedios caseros son los más utilizados durante el dolor menstrual y que ante la necesidad para mitigar el dolor el uso de antiespasmódicos es la norma seguido de los antiinflamatorios no esteroides sin predilección por ninguno. Cabe anotar que dentro de los tratamientos para el manejo de la dismenorrea grado I y III fuera de los aines (3031) los anticonceptivos orales son sustancias que han mostrado su utilidad en la dismenorrea (32) y en nuestra encuesta ninguna de las adolescentes lo mencionó. En nuestro medio el prescribir anticonceptivos durante la adolescencia acarrea problemas de índole cultural y religioso. Esta encuesta abre una serie de interrogantes que nos obliga a continuar su estudio.

\section{BIBLIOGRAFIA}

1. Onatra W., Martínez P., Franco G. Dismenorrea en adolescentes. Estudio epidemiológico. Curso complicaciones médicas en ginecología. Memorias. Universidad Nacional de Colombia. Instituto Materno Infantil. Bogotá. Edit. Presencia. 1989; 54-61.

2. Yikorkala O., Dawood MY. New concepts in dysmenorrhea. Am. J. Obstet. Gynecol. 1978; 130: 833-847.

3. Widholm O., Kantero RL. Correlations of menstrual traits between adolescent girls and their mothers. Acta Obstet. Gynecol. Scand. (Suppl) 1971; 50: 30-36.

4. Giordano J. Pesquisa sobre dismenorreia en mulhueres entre 18-40 años. Instituto Paulista de Pesquisa de Mercado. Sao Paulo 1981.

5. Svennerud S. Dysmenorrhea and absenteeism. Acta. Obstet. Gynecol. Scand. (Suppl) 1959; 38: 1-88.

6. Cambell H. World health organization. Multicenter study on menstrual an ovulatory patterns in adolescent girls. 1986; 7: 236-244.
7. Nuprin Pain. Report 1985.

8. Bergsjo P. Socioeconomic implications of dismenorrhea. Acta Obstet. Gynecol. Scand. (Suppl) 1979; 87: 67-68.

9. Chan WY., Dawood MY., Fuchs F. Prostaglandin in primary dysmenorrhea: comparison of prophylactic and no prophylactic treatment with ibuprofen and use of oral contraceptive. Am. J. Med. 1981; 70: 535-541.

10. Dawood MY. Monsteridal anti-inflammatory drugs and changing attitudes toward dysmenorrhea. Am. J. Med. 1988; (84): 23-29.

11. Cholst IN., Carlon AT. Oral contraceptives and dysmenorrhea. J. Adolesc. Health Care 1987; 8: 121-128.

12. Lawlor C., Davis AM. Primary dysmenorrhea: Relationship to prsonalyti and attitudes in adolescent females. J. Adolesc. Health Care 1981; 1: 208-212.

13. Bjorn A., Wilson I. An epidemiologic study of young women with dysmenorrhea. Am. J. Obstet. Gynecol. 1982; 144: 655-660. 
14. Dawood MY. Overall aproach to the management of dysmenorrhea. In Dawood MY, Ed. Dysmenorrhea Baltimore: Williams \& Wilkins, 1981; 261-279.

15. Jaramillo R. Adolescencia. Rev. Col. Obstet. Ginecol. 1984; 35: 401409.

16. Wilson C., Emans SJ et all. The relationships of calculated percent body fat, sports participation, age, and place of residence on menstrual patterns in healthy adolescent girls at an independent New England high school. Sexual Activ. Teeneagers 1988; 2: 163-168.

17. Sundell G., Wilson I., Anderesch B. Factors influencing the prevalence and severety of dysmenorrhea in young woman. Br. J. Obstet. Gynecol. 1990; 97: 588-594.

18. Metheny WP., Smith RP. The relationship among exercise, stress, and primary dysmenorrhea. J. Behav. Med. 1989; 12: 569-586.

19. Lee KA., Rittenhouse CA. Prevalence of perimenstrual symptons in employed women. Women health 1991; 17: 17-32.

20. Muse KN. Cyclic Pelvic Pain. Obstet. Gynecol. Clin. North. Am. 1990; 17: 427-440.

21. Avant RF. Dysmenorrhea. Prim Care 1988; 15: 549-559.

22. Johnson J. Level of knowledge among adolescent girls regarding effective treatment for dysmenorrhea. J. Adolesc. Health Care 1988; 9: $398-402$

23. Cronje HC., Kritzinger IE. Menstruation: Symptons, management and attitudes in university students. Int. J. Obstet. Gynecol. 1991; 35: 147-150.
24. Barros ACS., Takemoto AK., Coronado MR y Col. Rev. Iatros 1987; 6: 13-19.

25. Silverg JL., Martin NG. Genetic and environmental factors in primary dysmenorrhea and its relationship to anxiety depression and neuroticism. Behav. Genet. 1987; 17: 363-383.

26. Jones DY. Influence of dietary fat on self-reported menstrual symptoms. Physiol. Behav. 1987; 40: 483-487.

27. Busch CM., Costa PT Jr Whitehead WE. Severe perimenstrual symptoms: prevalence and effects on absenteeism and health care seeking in a non-clinical sample. Women Health 1988; 14: 59-74.

28. Guliaeva NV., Luzina NL et all. The inhibition stage of lipid peroxidation during stress. Biull EKSP Med. 1988; 106: 660-663.

29. Teperi J., Rimpel M. Menstrual pain, health and behavior in girls. Soc. Sci. Med. 1989; 29: 163-169.

30. Chan WY., Fuchs F., Powell AM. Effects of naproxen sodium on menstrual prostaglandins and primary dysmenorrhea. Obstet. Gynecol. 1983; 61: 285-287.

31. Pulkkinen MO., Csapo AI. Effect of ibuprofen on menstrual blood prostaglandin synthetase inhibitors. Acta Obstet. Gynecol. Scand. 1979; 87: 39-42.

32. Hauksson A., Ektrom P. The influence of a combined oral contraceptive on uterine activity and reactivity to agonists in primary dysmenorrhea. Acta Obstet. Gynecol. Scand. 1989; 68: 31-34.

\section{EVENTOS}

XIV CONGRESO LATINOAMERICANO DE OBSTETRICIA Y GINECOLOGIA

XIX CONGRESO CENTROAMERICANO DE OBSTETRICIA Y GINECOLOGIA FLASOG 93 Noviembre 28 a Diciembre 3 - Centro de Convenciones Atlapa - Panamá, Informes Federación Colombiana de Sociedades de Obstetricia y Ginecología. Tels. 2681485 - 2444692 Ext. 22

CONGRESO MUNDIAL DE ENDOSCOPIA GINECOLOGICA del 2 al 5 de diciembre de 1993 en Bombay, India. Informes Telfaz No. 91-22-3871415 Tel. 351522, 3885127 en Bombay.

IV CONGRESO MUNDIAL DE ENDOMETRIOSIS del 25 al 28 de mayo de 1994 en Salvador Baha Brazil. Informes Secretaría Congreso Rua Caetano Moura, 35 - Federacao, 40210-431 - Salvador Tel. (55-71) 247-0350.

\section{CONGRESO NACIONAL DE OBSTETRICIA Y GINECOLOGIA}

\section{Marzo 2 al 5 de 1994 Centro de Convenciones Gonzalo Jiménez de Quesada, Santafé de Bogotá.} VALOR INSCRIPCIONES

\begin{tabular}{llrlr} 
& \multicolumn{2}{c}{ FEBRERO $26 / 94$} & & \multicolumn{2}{c}{ CONGRESO } \\
FECOLSOG & $\$$ & 88.000 .00 & & $\$ 20.000 .00$ \\
NO MIEMBROS & $\$$ & 108.000 .00 & $\$$ & 140.000 .00 \\
RESIDENTES & $\$$ & 50.000 .00 & $\$$ & 65.000 .00 \\
EXTRANJEROS & US\$ & 150.00 & US\$ & 150.00
\end{tabular}

Para los Miembros de SCOG al día en las cuotas de sostenimiento se concede un $10 \%$ de descuento CURSOS DE POST-GRADO PRECONGRESO

ENDOCRINOLOGIA GINECOLOGICA

PATOLOGIA DEL TRACTO GENITAL INFERIOR

MEDICINA MATERNO FETAL

FECHA: $\quad$ Febrero 28 a marzo 1 de 1984

VALOR CURSO: $\$ 60,000.00$

Descuento del $20 \%$ para inscritos previamente en el CONGRESO

INFORMES E INSCRIPCIONES: Cra. 23 No. 39 - 82 Tel.: 2681485 - Telefax: 2444419 ó consignar en Bancoquia Cta. Cte. 04043921-8; Conavi Cta. No. 2055-010409758 